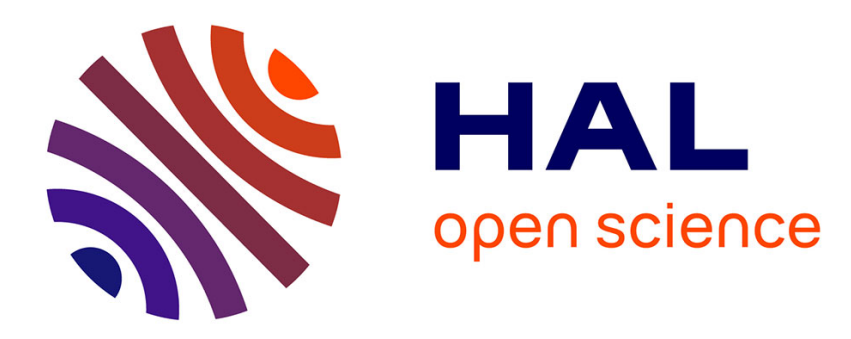

\title{
Spectre raman du sulfate de potassium
}

M. Debeau

\section{- To cite this version:}

M. Debeau. Spectre raman du sulfate de potassium. Revue de Physique Appliquée, 1972, 7 (1), pp.49-53. 10.1051/rphysap:019720070104900 . jpa-00243596

\section{HAL Id: jpa-00243596 https://hal.science/jpa-00243596}

Submitted on 1 Jan 1972

HAL is a multi-disciplinary open access archive for the deposit and dissemination of scientific research documents, whether they are published or not. The documents may come from teaching and research institutions in France or abroad, or from public or private research centers.
L'archive ouverte pluridisciplinaire HAL, est destinée au dépôt et à la diffusion de documents scientifiques de niveau recherche, publiés ou non, émanant des établissements d'enseignement et de recherche français ou étrangers, des laboratoires publics ou privés. 


\title{
SPECTRE RAMAN DU SULFATE DE POTASSIUM
}

\author{
M. DEBEAU \\ Département des Recherches Physiques (*), Université de Paris VI, 11, quai Saint-Bernard, Paris (Ve)
}

(Reçu le 13 décembre 1971)

\begin{abstract}
Résumé. - Le spectre Raman d'un monocristal de sulfate de potassium $\mathrm{K}_{2} \mathrm{SO}_{4}$ a été enregistré, à la température ordinaire, avec un spectrophotomètre équipé d'un laser à hélium-néon. Les bandes sont attribuées sans ambiguïté aux types de vibration $\mathbf{A}_{\mathrm{g}}, \mathbf{B}_{1 \mathrm{~g}}, \mathbf{B}_{2 \mathrm{~g}}$ et $\mathbf{B}_{3 \mathrm{~g}}$. L'influence de l'entourage des ions sulfate sur leurs modes internes de vibration est mise en évidence, ainsi que le mélange des modes de libration et de translation.
\end{abstract}

Abstract. - The laser-Raman spectrum of a single crystal of potassium sulphate $\mathrm{K}_{2} \mathrm{SO}_{4}$ has been measured at room temperature. From polarization measurements, bands are unambiguously assigned to the $A_{g}, B_{1 g}, B_{2 g}$ and $B_{3 g}$ types of vibration. The environment of sulphate ions influences their internal modes of vibration. The lattice modes are neither pure rotational nor pure translational.

Introduction. - Le spectre Raman du sulfate de potassium a déjà été étudié par plusieurs auteurs, soit sur des monocristaux non orientés [2], [7], soit en solution dans l'eau [1], [4], [5], soit encore sur des poudres [3], [6]. En solution aqueuse, quatre raies ont été observées : $981 \mathrm{~cm}^{-1}\left(v_{1}\right.$; forte et polarisée), $456 \mathrm{~cm}^{-1}\left(v_{2}\right.$; faible et dépolarisée $), 1102 \mathrm{~cm}^{-1}$ $\left(v_{3}\right.$; très faible et dépolarisée) et $617 \mathrm{~cm}^{-1}\left(v_{4}\right.$; faible et dépolarisée). Lorsque les expériences étaient faites sur des poudres ou des monocristaux, des dédoublements de fréquence ont été signalés pour toutes les raies, sauf pour $v_{1}$.

Dans tous ces travaux, il n'est question que des vibrations internes de l'ion sulfate. La multiplicité des bandes, qui apparaît croissante à mesure que les techniques expérimentales s'améliorent, indique une déformation du tétraèdre de l'ion sulfate, qui devient irrégulier dans le réseau cristallin.

L'examen, en diffusion Raman, d'un monocristal orienté a été repris pour tenter de définir plus précisément l'influence du champ cristallin sur les ions sulfate et pour déterminer les fréquences des vibrations propres au réseau.

Structure. - De nombreux auteurs se sont intéressé à la structure de $\mathrm{K}_{2} \mathrm{SO}_{4}$ [8], [9], [10]. Leurs résultats diffèrent peu les uns des autres. $\mathrm{K}_{2} \mathrm{SO}_{4}$ est orthorhombique et cristallise dans le groupe Pmcn $\left(D_{2 h}^{16}\right)$. Il y a quatre molécules par maille élémentaire. Les paramètres de la maille sont : $a=5,776 \pm 0,005 \AA$;

(*) Laboratoire associé au CNRS, $\mathrm{n}^{\circ} 71$. $b=10,08 \pm 0,01 \AA ; \quad c=7,456 \pm 0,006 \AA$. Les distances $\mathrm{S}-\mathrm{O}$ dans le tétraèdre $\mathrm{SO}_{4}^{2-}$ varient de

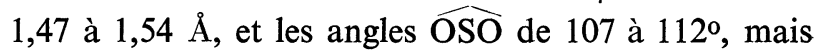
l'incertitude sur ces nombres ne permet pas d'affirmer l'irrégularité du tétraèdre. Les atomes de soufre sont dans des sites de symétrie $\mathrm{C}_{\mathrm{s}}$. Les atomes d'oxygène appartiennent à trois familles distinctes, dont deux ont une symétrie de site $\mathrm{C}_{\mathrm{s}}$ et une, la symétrie de site $\mathrm{C}_{1}$; les ions potassium appartiennent à deux familles de site $\mathrm{C}_{\mathrm{s}}$. Les plans de site sont perpendiculaires à l'axe $a$ et seront notés $\sigma(y z)$ dans la suite. La figure 1 représente la projection de la maille sur un de ces plans. Les atomes d'une même famille se correspondent par un centre d'inversion, ainsi que par des axes binaires avec glissement et des plans avec glissement.

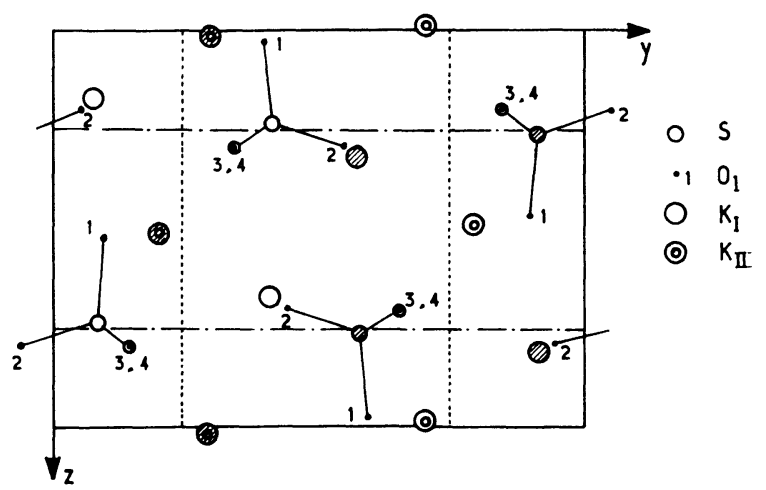

FIG. 1. - Projection de la maille cristallographique de $\mathrm{K}_{2} \mathrm{SO}_{4}$ sur un plan perpendiculaire à l'axe $a$. Les cercles hachurés sont les atomes situés dans le plan parallèle au plan de figure, à l'altitude $a / 2$. 
Dénombrement des vibrations. - L'ion sulfate, qui a la symétrie $T_{d}$ à l'état libre, se trouve donc en un site $\mathrm{C}_{\mathrm{s}}$; cet abaissement important de symétrie entraîne une cessation complète de la dégénérescence de ses modes fondamentaux de vibration. De plus, la présence de quatre molécules dans la maille provoque une multiplication du nombre des vibrations et l'existence d'un centre d'inversion conditionne leur activité : seules les vibrations symétriques par rapport au centre sont actives en Raman.

Les vibrations internes de l'ion sulfate libre sont $1 \mathrm{~A}_{1}, 1 \mathrm{E}$ et $2 \mathrm{~F}_{2}$. Le dénombrement des vibrations dans le cristal se fait à l'aide du tableau de corrélation (Tableau I), établi suivant les méthodes usuelles. Le tableau I donne en outre le classement des modes externes.

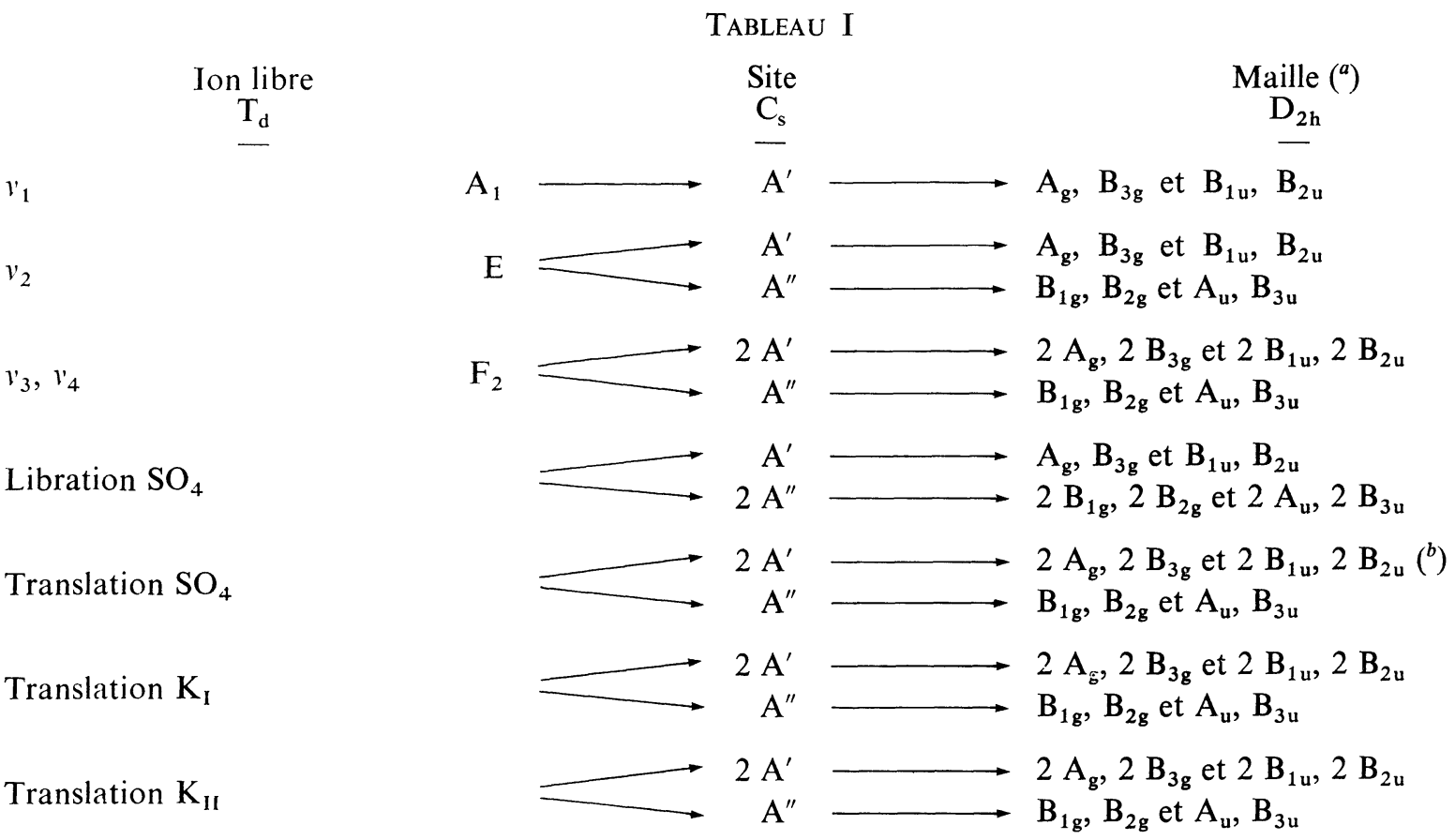

$\left({ }^{a}\right)$ Les vibrations $g$ sont actives en Raman, les vibrations $u$ actives en infrarouge, sauf les $A_{u}$ qui sont inactives.

(b) Au dénombrement des modes externes de translation, il convient de retrancher la translation de la maille rigide soit $1 \mathrm{~B}_{1 \mathrm{u}}, 1 \mathrm{~B}_{2 \mathrm{u}}, 1 \mathrm{~B}_{3 \mathrm{u}}$.

Les coefficients non nuls des tenseurs de polarisabilité dérivés pour les différents types de raies dans le groupe fini $\mathrm{D}_{2 \mathrm{~h}}$, sont les suivants : $\mathrm{A}_{\mathrm{g}}\left(\varepsilon_{x x}, \varepsilon_{y y}, \varepsilon_{z z}\right)$; $\mathrm{B}_{1 \mathrm{~g}}\left(\varepsilon_{x y}\right) ; \mathrm{B}_{2 \mathrm{~g}}\left(\varepsilon_{x z}\right) ; \mathrm{B}_{3 \mathrm{~g}}\left(\varepsilon_{y z}\right)$.

Résultats expérimentaux. - La lumière incidente polarisée, la lumière diffusée analysée et une orientation convenable, dans le montage, d'un monocristal taillé de $\mathrm{K}_{2} \mathrm{SO}_{4}$, permettent d'attribuer sans ambiguité les raies observées aux différents types de symétrie. Le monocristal utilisé a été taillé [11] en forme de parallélépipède rectangle de $4 \times 4 \times 10 \mathrm{~mm}^{3}$, dont une paire de faces est perpendiculaire à la bissectrice $y$ de l'angle obtus ( $y$ est parallèle à $b$ ), une autre perpendiculaire à la bissectrice $z$ de l'angle aigu des axes $(z \| c)$, et la troisième, normale à $x(x \| a)$, est parallèle au plan des axes optiques.

Les spectres ont été enregistrés avec un spectrophotomètre Coderg PH 1, équipé d'un laser à héliumnéon de Spectra Physics ( $\simeq 35 \mathrm{~mW}$ sur le cristal). La figure 2 représente l'enregistrement de quelques spectres. Le tableau II montre les nombres d'onde, à $2 \mathrm{~cm}^{-1}$ près, observés dans les différents cas consi- dérés, correspondant chacun à un des coefficients des tenseurs de polarisabilité dérivés. Les intensités indiquées sont des intensités relatives, estimées par la mesure au planimètre de la surface des bandes. Les raies externes, de fréquences inférieures à $200 \mathrm{~cm}^{-1}$, sont d'intensité trop faible pour se prêter aux mesures.

Interprétation et discussion. - 1) VIBRATIONS INTERNES. - Si, d'abord, on ne tient pas compte du couplage entre les ions, à chaque vibration d'un ion dans le cristal, issue d'une vibration de l'ion libre, correspond une coordonnée normale, représentée par un vecteur situé, soit dans le plan de symétrie si la vibration est de type $A^{\prime}$, soit perpendiculairement à ce plan si la vibration est de type $A^{\prime \prime}$.

Des considérations théoriques, à l'approximation d'ordre zéro qui utilise les tenseurs de l'ion libre, ont été développées par Couture [12] pour des cristaux de même groupe spatial que le sulfate de potassium. Elles conduisent à la prédiction de l'intensité des raies dans le groupe $D_{2 h}$, compte tenu de l'orientation des ions sulfate dans le cristal ; cette orientation est repérée par l'angle $\alpha$ formé par l'axe binaire $\mathrm{Su}$ de 


\section{TABLEAU II}
$\mathrm{A}_{g}\left(\varepsilon_{x x}^{2}\right)$
$\mathrm{A}_{\mathrm{g}}\left(\varepsilon_{y y}^{2}\right)$
$\mathrm{A}_{\mathrm{g}}\left(\varepsilon_{z z}^{2}\right)$
$\mathrm{B}_{38}\left(\varepsilon_{y_{z}}^{2}\right)$
$\mathrm{B}_{1 \mathrm{~g}}\left(\varepsilon_{x y}^{2}\right)$
$\mathbf{B}_{2 \mathrm{~g}}\left(\varepsilon_{x z}^{2}\right) \quad$ Attribution

Fréq. ${ }^{\text {Int. Fréq. }}$ Int. Fréq. Int. Fréq. Int. Fréq. Int. Fréq. Int. proposée

$\begin{array}{llllll}1 \overline{144} & -2 & 1 \overline{143} & - & 1 \overline{145} & \overline{59}\end{array}$

$\begin{array}{llll}1092 & 13 & 1091 & 21\end{array}$

$\begin{array}{llll}983 & 123 & 983 & 180\end{array}$

$\begin{array}{llll}627 & 3 & 629 & 25\end{array}$

$983-190 \quad 1111 \quad 12$

$1104 \quad 46 \quad 1109$

$19\} v_{3}$

$\begin{array}{lllllll}983 & 6 & 983 & 5 & 983 & 6 & v_{1}\end{array}$

$\begin{array}{rrr}983 & 190 & 983 \\ 628 & 50 & 633\end{array}$

$\begin{array}{llll}618 & 23 & 617 & 42\end{array}$

$620 \quad 6$

$623 \quad 51$

622

$620 \quad 24$

$457 \quad 52$

456

$453 \quad 88$

140

160

138

Vibrations

$130 ?$

131

$135 ?$

118

111

111

109

109

de

100

94

91
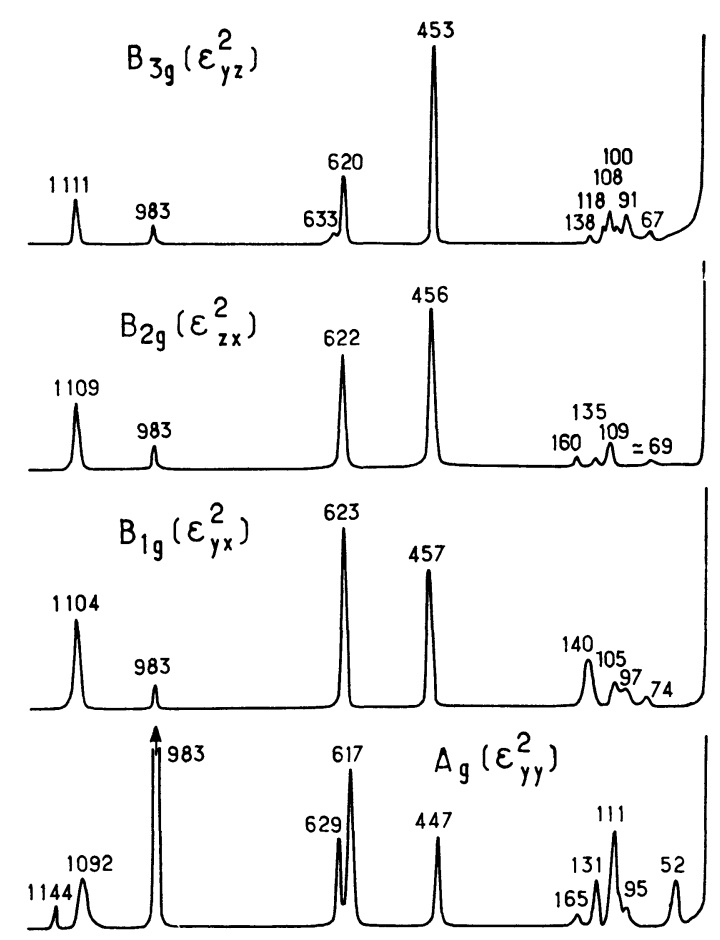

FIG. 2. - Spectres correspondant aux divers types de raies. Les fréquences sont exprimées en $\mathrm{cm}^{-1}$. l'ion libre, situé dans le plan $\sigma(y z)$ et l'axe $y$ (cf. Fig. 3).

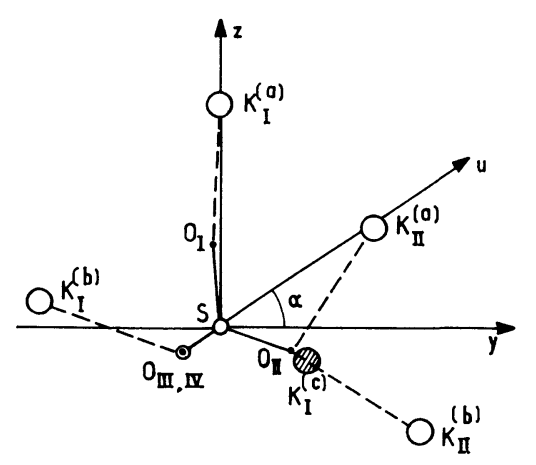

FIG. 3. - Entourage d'un ion sulfate dans le plan $\sigma(y z)$. Le cercle hachuré représente les ions $\mathrm{K}^{+}$situés dans le plan parallèle au plan de figure, à l'altitude $\pm a / 2$.

$-v_{1}$. Les intensités pour les raies $\mathrm{A}_{\mathrm{g}}$ et $\mathrm{B}_{3 \mathrm{~g}}$ permises par la théorie des groupes et issues de la vibration $v_{1}$ totalement symétrique de l'ion libre, sont théoriquement, à l'approximation d'ordre zéro, pour $\mathrm{A}_{\mathrm{g}}: \varepsilon_{x x}^{2}=\varepsilon_{y y}^{2}=\varepsilon_{z z}^{2}$ et pour $\mathrm{B}_{3 \mathrm{~g}}: \varepsilon_{y z}^{2}=0$. La raie $983 \mathrm{~cm}^{-1}$ est attribuée à cette vibration. Elle se 
retrouve dans tous les spectres avec des intensités très inégales. $\varepsilon_{y y}^{2}$ est à peu près égal à $\varepsilon_{z z}^{2}$ et $\varepsilon_{x x}^{2}$ est plus faible. Cette différence reflète le fait que l'ion n'est plus isotrope dans le cristal et qu'il est influencé différemment par son entourage dans le plan $\sigma(y z)$ et hors du plan.

L'approximation d'ordre zéro n'est pas tout à fait valable et c'est bien ce qui singularise $\varepsilon_{x x}$. La raie apparaît faiblement dans les spectres $B_{1 \mathrm{~g}}$ et $B_{2 \mathrm{~g}}$ où elle est interdite. Une légère incertitude dans la polarisation de la lumière ou dans l'orientation du cristal suffit à expliquer sa présence, étant donnée sa très forte intensité intrinsèque. Dans le spectre $B_{3 g}$ où elle est permise, la raie n'apparaît pas plus forte que dans les spectres $B_{1 \mathrm{~g}}$ et $\mathrm{B}_{2 \mathrm{~g}}$. Sa présence peut être attribuée, soit à une incertitude de polarisation et d'orientation comme précédemment, soit au fait que l'approximation d'ordre zéro, à laquelle les calculs sont faits, peut être trop grossière et que, alors, $\varepsilon_{y z}^{2}$ peut n'être pas nul, bien que gardant une valeur très faible.

- $v_{2}$. Les raies $447,453,456$ et $457 \mathrm{~cm}^{-1}$ sont attribuées respectivement aux vibrations $A_{g}, B_{3 g}, B_{2 g}$ et $B_{1 \mathrm{~g}}$ issues du mode $v_{2}$ doublement dégénéré de l'ion libre. L'abaissement de symétrie décompose celui-ci en une vibration $\mathrm{A}^{\prime \prime}$ antisymétrique et une vibration $\mathrm{A}^{\prime}$ symétrique par rapport au plan $\sigma(y z)$. La variation théorique du carré des coefficients des tenseurs de polarisabilité dérivés correspondant à ces vibrations, en fonction de l'angle $\alpha$ (angle $\mathrm{Su}, y$ ) est donnée par les formules :

$$
\begin{aligned}
& \varepsilon_{x x}^{2}=1 \\
& \varepsilon_{y y}^{2}=\left(\sin ^{2} \alpha-2 \cos ^{2} \alpha\right)^{2} \\
& \varepsilon_{z z}^{2}=\left(\cos ^{2} \alpha-2 \sin ^{2} \alpha\right)^{2} \\
& \varepsilon_{x y}^{2}=3 \sin ^{2} \alpha \\
& \varepsilon_{x z}^{2}=3 \cos ^{2} \alpha \\
& \varepsilon_{y z}^{2}=9 \sin ^{2} \alpha \cos ^{2} \alpha .
\end{aligned}
$$

D'après les données cristallographiques, $\alpha \simeq 32^{\circ}$. Pour que la comparaison entre les intensités théoriques et expérimentales soit possible, l'intensité de la raie $A_{g}$ correspondant au coefficient $\varepsilon_{x x}^{2}$ est prise égale à 1 et celle des autres raies est calculée par rapport à celle-ci. Le tableau III présente les nombres obtenus

\begin{tabular}{|c|c|c|c|c|c|c|}
\hline & - & - & - & - & - & - \\
\hline Int. prévues & 1 & 1,34 & 0,02 & 0,84 & 2,16 & 1,82 \\
\hline Int. observées & 1 & 1,37 & 0 & 3,25 & 2,19 & 5,5 \\
\hline
\end{tabular}
ainsi. La concordance entre les intensités calculées et observées est bonne pour les coefficients des vibrations $A_{g}$ et $B_{2 g}\left(\varepsilon_{x z}^{2}\right)$. Par contre, les raies $B_{1 g}$ et $\mathbf{B}_{\mathbf{3 g}}$ sont beaucoup plus intenses que prévu. Autre-

\section{TABLeAU III}

ment dit, si les fréquences des modes normaux issus de $v_{2}$ sont voisines de ce qu'elles sont dans l'ion libre (la cessation de dégénérescence et le couplage entre les ions n'entraînent qu'un écart de $9 \mathrm{~cm}^{-1}$ ), leurs variations de polarisabilité sont différentes.

La figure 3 montre l'entourage immédiat d'un ion sulfate. Quatre des plus proches atomes de potassium sont dans le plan $\sigma(y z)$ qui est le plan de figure ; les deux autres, dont les projections se confondent dans la figure avec le cercle hachuré $K_{\mathrm{I}}^{(\mathrm{c})}$ sont dans les plans parallèles de cotes $\pm a / 2$. Les traits pointillés relient chaque atome de potassium à l'atome d'oxygène le plus proche. Les distances $\mathrm{K}-\mathrm{O}$ varient de 2,64 à $2,90 \AA$. Si $K_{\mathrm{I}}^{(\mathrm{a})}, K_{\mathrm{II}}^{(\mathrm{a})}$ et $K_{\mathrm{II}}^{(\mathrm{b})}$ se placent à peu près symétriquement par rapport à $\mathrm{Su}$, il n'en est pas de même pour $K_{\mathrm{I}}^{(\mathrm{b})}$ et les deux $K_{\mathrm{I}}^{(\mathrm{c})}$. Ces trois derniers atomes sont placés près de l'axe $\mathrm{S} y$. Les coefficients des tenseurs de polarisabilité dérivés correspondant aux vibrations non totalement symétriques et ayant $y$ en indice, sont affectés par cette disposition particulière des cations autour de l'ion sulfate. Le rapport entre les valeurs calculées des intensités et les valeurs observées pour les modes $\mathbf{B}_{\mathbf{1 g}}$ et $\mathbf{B}_{\mathbf{3 g}}$, est une indication de l'importance de l'influence des ions potassium sur le mode $v_{2}$. Dans la mesure où certains cations paraissent jouer un rôle spécial dans ce mode, une participation des modes externes n'est pas exclue.

$-v_{3}$ et $v_{4}$. L'abaissement de symétrie transforme un mode triplement dégénéré de l'ion libre en une vibration antisymétrique et en deux vibrations symétriques par rapport au plan $\sigma(y z)$. Le couplage entre les quatre ions de la maille multiplie ensuite les fréquences. Pour les vibrations issues des modes $v_{3}$ et $v_{4}$, les valeurs théoriques des coefficients $\varepsilon_{x y}^{2}$ et $\varepsilon_{x z}^{2}$ des tenseurs de polarisabilité sont [12] : $\left(\mathrm{B}_{1 \mathrm{~g}}\right) \varepsilon_{x y}^{2}=\cos ^{2} \alpha$; $\left(\mathrm{B}_{2 \mathrm{~g}}\right) \varepsilon_{x z}^{2}=\sin ^{2} \alpha$. Avec $\alpha=32^{\circ}$, le rapport $\varepsilon_{x y}^{2} / \varepsilon_{x z}^{2}$ vaut 2,57 . L'expression des autres coefficients contient, outre $\alpha$, un paramètre supplémentaire qui représente la façon dont cesse la dégénérescence dans le plan. Ce paramètre n'est pas fixé par les conditions cristallographiques, mais dépend du champ des forces interioniques.

Dans l'ensemble issu de $v_{3}$, cinq raies sont observées au lieu de six prévues : $1104 \mathrm{~cm}^{-1}\left(B_{1}\right)$ et $1109 \mathrm{~cm}^{-1}$ $\left(\mathrm{B}_{2 \mathrm{~g}}\right)$ correspondent à la vibration antisymétrique par rapport à $\sigma(y z)$. Ces deux vibrations ayant des fréquences voisines, nous admettrons qu'il en est de même pour les deux dérivées d'une vibration symétrique par rapport au plan, et nous associerons $1091 \mathrm{~cm}^{-1}\left(\mathrm{~A}_{\mathrm{g}}\right)$ et $1111 \mathrm{~cm}^{-1}\left(\mathrm{~B}_{3 \mathrm{~g}}\right)$ d'une part, $1144 \mathrm{~cm}^{-1}\left(\mathrm{~A}_{\mathrm{g}}\right)$ d'autre part (la raie $\mathrm{B}_{3 \mathrm{~g}}$ correspondante est absente). Le rapport des intensités de $B_{1 \mathrm{~g}}$ et de $B_{2 g}$ est 2,42 , valeur voisine de celle prévue. Leurs fréquences restent très voisines de leur valeur (1 $102 \mathrm{~cm}^{-1}$ ) dans l'ion libre, tandis que, dans le plan, les effets de champs statique et dynamique provoquent un important déplacement de fréquences : $53 \mathrm{~cm}^{-1}$ pour $A_{g}$. 
Les six raies attendues pour l'ensemble issu de $v_{4}$ sont observées : $623 \mathrm{~cm}^{-1}\left(\mathrm{~B}_{1 \mathrm{~g}}\right)$ et $622 \mathrm{~cm}^{-1}\left(\mathrm{~B}_{2 \mathrm{~g}}\right)$ viennent de la vibration antisymétrique par $\cdot$ rapport au plan ; $618 \mathrm{~cm}^{-1}\left(\mathrm{~A}_{\mathrm{g}}\right)$ et $620 \mathrm{~cm}^{-1}\left(\mathrm{~B}_{3 \mathrm{~g}}\right)$ d'une part, $628 \mathrm{~cm}^{-1}\left(\mathrm{~A}_{\mathrm{g}}\right)$ et $633 \mathrm{~cm}^{-1}\left(\mathrm{~B}_{3 \mathrm{~g}}\right)$ d'autre part, correspondent aux deux vibrations symétriques par rapport à $\sigma(y z)$. Le rapport des intensités $\varepsilon_{x y}^{2} / \varepsilon_{x z}^{2}$ est 2,13 , ce qui est encore relativement proche de la valeur théorique. Le couplage entre les ions est faible et les fréquences gardent des valeurs voisines de celles de l'ion libre.

2) Vibrations eXternes. - Le sulfate de potassium a de nombreuses vibrations de réseau. On attend $7 \mathrm{~A}_{\mathrm{g}}, 5 \mathrm{~B}_{1 \mathrm{~g}}, 5 \mathrm{~B}_{2 \mathrm{~g}}$ et $7 \mathrm{~B}_{3 \mathrm{~g}}$. Dans aucun spectre, n'ont été observées toutes les raies théoriquement permises. La raie $B_{2 g}$ à $69 \mathrm{~cm}^{-1}$ est douteuse.

Il est difficile de distinguer les raies de translation et les raies de libration. Un calcul élémentaire [13] suppose que les pivotements de l'ion se font autour des axes principaux d'inertie, que ceux-ci coïncident avec les axes du tenseur de polarisabilité et que ce dernier n'est pas modifié lorsque l'ion est introduit dans le cristal. Dans le cas du sulfate de potassium, un tel calcul ne donne pas de résultat convaincant: on ne peut trouver, dans les spectres, des raies dont les rapports d'intensité dans les différents cas d'observation, soient ceux calculés. Le tenseur de polarisabilité de l'ion dans son site est différent de celui de l'ion libre. En outre, une interaction entre les modes de translation et les modes de rotation peut modifier les intensités des raies.

Cette dernière remarque est corroborée si l'on considère les raies $B_{1 g}$ et $B_{2 g}$ : l'écart entre la fréquence d'une $B_{1 \mathrm{~g}}$ et celle de la $B_{2 \mathrm{~g}}$ correspondante, est important : entre 7 et $30 \%$. Le couplage des ions dans la maille n'est vraisemblablement pas suffisant pour donner lieu, seul, à une telle différence.

Conclusion. Le spectre Raman d'un monocristal de $\mathrm{K}_{2} \mathrm{SO}_{4}$ comprend de nombreuses bandes de vibration à cause de la cessation de dégénérescence due à la basse symétrie du cristal (champ statique) et du couplage entre les quatre ions de la maille (champ dynamique). Presque toutes les raies internes $\mathrm{de}_{4}^{2-}$ attendues ont été observées, leur type de symétrie a été défini et leur attribution faite. Il est difficile de séparer les effets dus au champ statique de ceux dus au champ dynamique, car les déplacements de fréquence constatés dans certains cas, reflètent l'influence simultanée de ces deux champs. L'intensité relative des raies est à peu près celle calculée à l'approximation d'ordre zéro ; il semble donc que l'ion sulfate soit assez peu déformé dans ce cristal.

De nombreuses raies, entre 160 et $50 \mathrm{~cm}^{-1}$, ont été attribuées aux vibrations de réseau; de fortes interactions font qu'il y a certainement peu de raies de libration ou de translation pures.

\section{Bibliographie}

[1] Nisi (H.), Jap. J. Phys., 1929, 5, 119.

[2] Nisi (H.), Jap. J. Phys., 1931, 7, 1.

[3] Krishnamurti (P.), Ind. J. Phys., 1930, 5, 183.

[4] Ramaswamy (C.), Ind. J. Phys., 1930, 5, 193.

[5] Cabannes (J.), Ann. Acad. Scient. Fennicae, Série AI, $1950,82,1$.

[6] Heerdt (R.) et Goubeau (J.), Z. anorg. allg. Chem., 1948, 255, 309.

[7] Riche (N.), DES Paris, 1948.

[8] Struktur Berichte, 2, 86 ; Structure Reports, 16, 284.
[9] Robinson (M. T.), J.'Phys. Chem., 1958, 62, 925 ; Structure Reports, 22, 447.

[10] Bernard (M.) et Hocart (R.), Bull. Soc. Franç. Minéral. Crist., 1961, 84, 396 ; Structure Reports, 26, 436.

[11] Taillé et poli par Mme Vernazza (E.)

[12] Couture (L.), Ann. Phys., 1947, 2, 5.

[13] Poulet (H.) et Mathieu (J. P.), Spectres de vibration et symétrie des cristaux, Gordon \& Breach, New York, 1970. 